\title{
TOWARDS INTEGRATED EARTH SENSING: THE ROLE OF IN SITU SENSING
}

\author{
P.M. Teillet ${ }^{1}$, R.P. Gauthier, and A. Chichagov \\ Canada Centre for Remote Sensing, 588 Booth Street, Ottawa, Ontario, K1A 0Y7, Canada
}

First International Workshop on Future Intelligent Earth Observing Satellites (FIEOS)

KEY WORDS: In Situ, Sensorweb, Assimilation, Wireless, Remote Sensing, Geospatial

\begin{abstract}
:
Significant advancements in Earth observation are expected to come about by developing more systematic capabilities for assimilating remote sensing observations and in situ measurements for use in models, at relevant scales, to generate geophysical and biospheric information products. This paper provides an overview of the role of in situ sensing in the context of integrated Earth sensing. It also defines a framework for taking advantage of intelligent sensorwebs based on the converging technologies of microsensors, computers, and wireless telecommunications in support of critical activities such as the monitoring of remote environments, risk assessment and hazard mapping, and renewable resource information management. The knowledge gleaned from integrated Earth sensing has the potential to empower managers and decision makers to act on critical climate, sustainable development, natural resource, and environmental issues. The paper also describes early work on a prototype wireless intelligent sensorweb evaluation in the context of watershed management and flood forecasting.
\end{abstract}

\section{INTRODUCTION}

Over the next few years, the nature of remote and in situ sensing and their relationship with the Internet will change drastically. Trillions of sophisticated embedded measuring devices will be networked together to provide an extensive monitoring system for the Earth. In parallel to these developments and those in satellite telecommunications, the future of Earth observation satellites is exemplified by NASA's strategic Earth science vision for networks of satellite sensors, or "sensorwebs". Much of the focus of these satellite sensor webs over the next 25 years will be on improving predictions of Earth system changes, both short-term and long-term, with considerable priority given to severe weather phenomena and disaster events (NASA, 2000). These orbital sensor deployments will provide the robustness that an operational system requires by allowing many small, separate platforms working together intelligently to accomplish what was formerly thought to require large, multi-sensor platforms.

From the rudimentary tools of past centuries to today's automated stations, field measurement instrumentation has usually kept pace with technological change. Today, utility companies make use of wearable computers and a variety of organisations including educational institutions take advantage of telepresence. A look ahead indicates that the converging technologies of microsensors, computers, and wireless telecommunications are creating new concepts such as in situ sensorwebs, which could have as much impact on the uses of sensor technology as the Internet did on the uses of computer technology.

This paper presents the concept of integrated Earth sensing, whose goal is to make significant advancements in the practical use of Earth observation data by developing intelligent in situ measurement capabilities that open new pathways towards the

\footnotetext{
${ }^{1}$ Corresponding author (phil.teillet@ccrs.nrcan.gc.ca).
}

generation of quantitative geophysical and biospheric information products. The concept encompasses advanced technologies to design and deploy intelligent sensor networks for in situ data acquisition, develop methods to assimilate in situ and remote sensing data into models that generate validated information products, and facilitate the accessibility of in situ sensor data and/or metadata from on-line geospatial data infrastructures. The paper outlines early work on the first of these and describes a Prototype Wireless Intelligent Sensorweb Evaluation (ProWISE). A logical data flow chart is presented to illustrate how specific information products will be developed in the context of watershed management and flood forecasting.

\section{DEFINITIONS}

\subsection{Remote Sensing}

Remote sensing can be defined as a technology used to acquire information about an object by detecting energy reflected or emitted by that object when the distance between the object and the sensor is much greater than any linear dimension of the sensor. A short dictionary-based definition could be that remote sensing is "sensing from a great distance". For the present purposes and in practice, it is the gathering of data about the Earth and environment by satellite sensors.

\subsection{In Situ Sensing}

In situ sensing can be defined as a technology used to acquire information about an object when the distance between the object and the sensor is comparable to or smaller than any linear dimension of the sensor. A short dictionary-based definition for in situ sensing could be "sensing in place". Because many measurements or observations are made from nearby locations that are not strictly speaking in situ, the 
expression proximal sensing has been adopted in a variety of disciplines. A short dictionary-based definition could be "sensing from close range" (as in close-range photogrammetry, for example). For the present purposes and in practice, in situ sensing is considered to encompass proximal sensing. Networks of in situ sensors have been in place for decades in numerous of contexts, perhaps the most prevalent being meteorological stations. These networks continue to evolve as unattended sensor and wireless telecommunication technologies advance at a rapid pace and new uses are invented. It is becoming increasingly feasible to provide quality-controlled network-wide data to users via the Internet in near real time and information products from data fusion and assimilation into models within hours.

\subsection{Data Fusion and Data Assimilation}

The following definitions were extracted from detailed material found on the Internet and are presented as being reasonably straightforward ones.

Data fusion is the seamless integration of data from disparate sources. The data have been integrated across data collection platforms and geographic boundaries, and blended thematically, so that the differences in resolution and coverage, treatment of a theme, character and artefacts of data collection methods are eliminated. At present, this is a desirable but very difficult goal to attain.

Data assimilation integrates observations and model predictions to provide a best estimate of the state of a system (i.e., observations help to keep the model "on track"). Broadly, the expectations of a data assimilation system are to:

- Organize the observations from diverse sources with heterogeneous space and time distribution into a regularly gridded, time continuous product.

- Complement the observations by propagating information from observed to unobserved regions.

- Supplement observations by producing estimates of unobserved quantities, using the model parameterizations constrained directly and indirectly by the observations.

- Maximize the physical and, ultimately, chemical consistency between the observations through the comprehensive parameterizations of the model.

- Provide a variety of quality control functions for the observations.

- Provide, ultimately, an instrument calibration capability, especially with regard to the definition of biases and instrument drift.

\section{INTEGRATED EARTH SENSING}

Satellite Earth observation sensors provide unique measurements of geophysical and biospheric variables globally and repetitively. These measurements are all the more critical because the Earth as a system changes constantly over a wide range of temporal and spatial scales. Nevertheless, it has long been recognized that ground data collection is an essential source of information even in surveys that rely heavily on remote sensing (Pettinger, 1971; Lee, 1975; Justice and Townshend, 1981; Teillet, 1995). With the increasing availability of multiple satellite sensor systems, it is essential to provide ground-based benchmarks and cross-calibration standards on a continuous basis to ensure the self-consistency of information products generated from these multiple systems (Teillet et al, 2001a).

After thirty years of research and development and a notable under-utilisation of information products based on remote sensing by government agencies and resource developers, it is now widely acknowledged that pixel signals as measured by Earth observation sensors do not contain all of the information necessary to derive many of the geophysical and biospheric parameters required to address current issues of critical importance to citizens.

Hence, a growing perspective today is that significant advancements in Earth observation are expected to come about only by developing more systematic capabilities for the fusion of remote sensing observations and in situ measurements for assimilation with models, at relevant scales, to generate geophysical and biospheric information products. This integrated approach will include the specification and deployment of unattended intelligent sensorwebs in various application contexts. Projects should be undertaken to demonstrate the importance and impact of the fusion of in situ measurements of quantities such as spectral reflectance, temperature, soil moisture, dielectric constant, etc., with remotely sensed pixel signals for use in relevant multi-scale models for the production of forecasts, yields, or hazard potential, among many others.

Earth observation agencies need to develop new data acquisition strategies and systems for integrated Earth sensing to monitor remote environments, hazards and disasters, and natural resources. Such an integrated Earth sensing capability can provide essential validated information for decision making if it involves interagency cooperation, common data processing standards, and timely access to data and information products on a long-term basis.

\section{EMERGING TECHNOLOGIES}

\subsection{Wearable Computers}

Field campaigns that take advantage of real-time data uplink and downlink to/from a common geospatial gateway from any point on Earth and at any given time will soon become commonplace. In years to come, in those cases where field research will still require a physical human presence, people will no longer have to carry computers, sensors and instruments to the field. They will be embedded or worn in clothes and other paraphernalia. Wearable computers and clothing-based multimedia computers are novel terms in our vocabulary and yet, today, these devices already exist (Mann, 1997) and are capable of exchanging data, voice and video, as well as providing accurate viewpoints in real-time while processing and analysing field data. Wearable computers are currently being used operationally by utility companies and experimentally in the Houghton Mars Project, for example, a NASA-led international field research program dedicated to planning the exploration of the planet Mars.

\subsection{Telepresence}

Advances in video technology have opened the door for telepresence, the capability of being present in a real-time sensory way at locations remote from one's own physical 
location (Buxton, 1997). Investigators undertaking field campaigns will be able to communicate and exchange their results and ideas with colleagues at other locations in real-time. This will contribute to standardization of measurement methodologies and protocols, remote technical support of instruments, lower-cost and wider participation in resourceintensive activities, university-level education, and training of highly qualified personnel (Teillet et al., 2001a). Following posted schedules, live webcasting and telepresence will provide experiment-oriented or pedagogical coverage of the in situ measurement activities. Issues and controversies about measurements and methodologies can be featured during broadcasts.

\subsection{Sensor Pods, Sensorwebs, and Smart Dust}

The automation of field mensuration is increasingly removing the operator from the field, particularly in hazardous environments. It is indeed becoming possible to remotely control and monitor field activities through the Internet. In particular, the converging technologies of sensors, computers, and telecommunications have created the new instrument concept of intelligent sensorwebs (Delin and Jackson, 2000, 2001). As opposed to other distributed sensor networks, sensors in a sensorweb share information among themselves and modify their behaviour on the basis of collected data. In the in situ sensing context, a sensorweb consists of wirelessly communicating, spatially distributed sensor pods that are deployed to monitor and explore environments (Figure 1). The pods serve as nodes in a communication chain or network that links up to base stations for wireless transmission to a user infrastructure. They can also have plug-in capability to handle data from other environmental sensors. With the capability of providing an ongoing virtual presence in remote locations, many sensorweb uses can be envisaged in the context of environmental monitoring. Sensorwebs could have as much impact on the uses of sensor technology as the Internet did on the uses of computer technology.

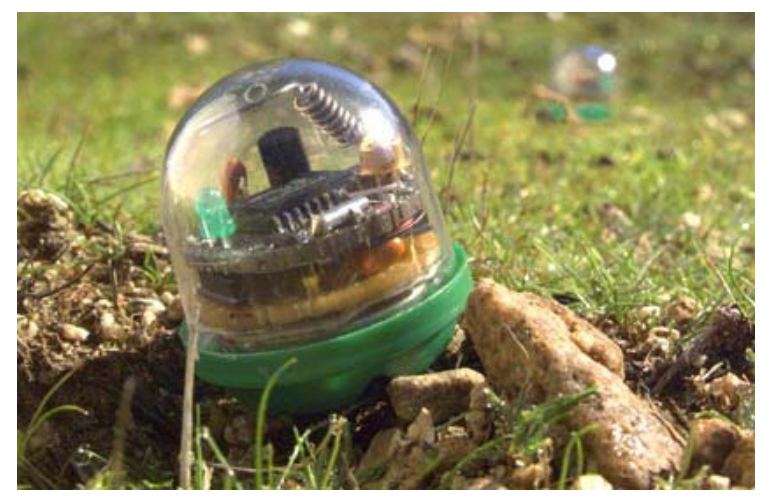

Figure 1. A sensor pod from the Jet Propulsion Laboratory (JPL).

Already being investigated, the next step will be Smart Dust sensing, computation, communication, and power in a cubic millimetre - deployed in large numbers with the ability to measure temperature, pressure, light, sound, humidity, magnetic field, and acceleration (Pister et al., 1999). Many uses are being considered, but an example would be forest fire warning and monitoring. Quickly developed prototypes have been built using commercial-off-the-shelf (COTS) components. COTS
Dust has the same functionality as Smart Dust, but the devices are a cubic inch in size instead (Hollar, 2000).

\subsection{Global Virtual Presence}

An emerging perspective is that the advent of in situ sensorwebs, Earth science satellite sensorwebs, and the Internet will provide a kind of global virtual presence, as described by NASA's Jet Propulsion Laboratory (JPL) (Figure 2) or, alternatively, integrated Earth sensing. There is great potential for applying existing and emerging in situ sensor technologies and networks in order to achieve the goals of resource and environmental monitoring activities. The criteria for success will be a dramatic increase in the efficiency of data collection and analysis, as well as the timely availability of data and derived information for decision-making.

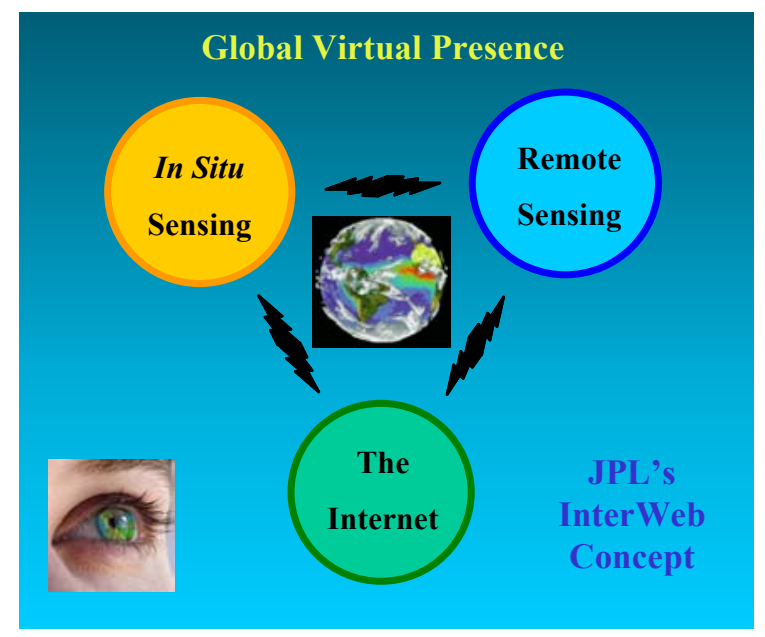

Figure 2. Schematic diagram adapted from JPL's InterWeb concept (Delin and Jackson, 2001).

\section{A FRAMEWORK FOR IN SITU SENSING ACTIVITIES}

Because numerous independently managed networks and archives of in situ sensors and data currently exist, it will be important to focus activities carefully and leverage existing infrastructures wherever possible. It is proposed that, initially, in situ sensor measurement assimilation activities fit within the following framework (Teillet et al., 2001b) (Figure 3). They should:

- Lead to the generation of geophysical and biospheric information products that address clearly defined science and technology questions and/or user information requirements.

- Utilize in situ sensor networks.

- Utilize remote sensing data products.

- Encompass data assimilation and validation components.

- Routinely provide in situ data products and/or metadata on in situ data holdings to a geospatial data infrastructure.

In situ sensor measurement assimilation activities could focus as a priority on issue-driven science and technology activities such as the monitoring of remote environments, risk assessment and hazard mapping, and time-critical decision making (e.g., disaster and renewable resource information management). For 
instance, Figure 4 illustrates the framework in the context of watershed management. Additional examples are the applications prototypes developed by Wood et al. (2002) and Hochheim et al. (2002) in the contexts of flood information management and crop specific projections, respectively.

Complementary to current efforts towards an Integrated Global Observing Strategy ${ }^{2}$, the integrated Earth sensing concept will provide a framework for the research and development of advanced data acquisition and integration elements of environmental monitoring/information systems used for local, regional, or global decision-making.

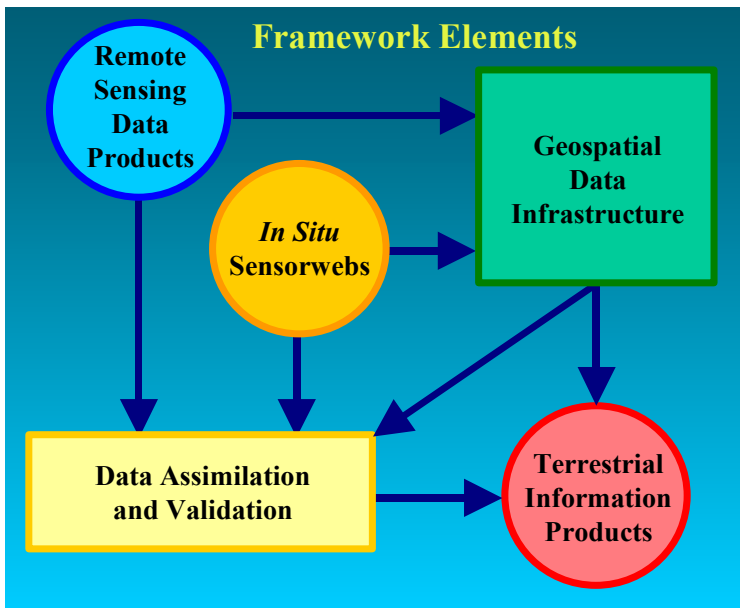

Figure 3. Principal framework elements for in situ sensor measurement assimilation activities.

\section{PROTOTYPE WIRELESS INTELLIGENT SENSORWEB EVALUATION (PROWISE)}

A technology test-bed is necessary to integrate the various components of a sensorweb so that it can be remotely controlled and eventually remotely configured. The process involves solving the engineering problems associated with the interfaces between the sensors at a given node and the wireless telecommunications devices that will include the addition of control and decision drivers (machine intelligence). Field trials are required to evaluate deployment and remote operation issues as well as considerations relevant to the use of sensorwebs in specific information extraction and decision contexts. The objectives of the Prototype Wireless Intelligent Sensorweb Evaluation (ProWISE) activity currently underway will involve two to three field seasons.

\subsection{Objective 1: Deploy, Activate, and Remotely Access/Control the Sensorweb}

This will eventually involve the field deployment of a sensorweb with full internodal connectivity and remote access and control. Initially, a prototype network of sensors has been deployed in a controlled outdoor environment in the Ottawa area to facilitate the debugging of the protocol conversion between the microsensor/microcontroller packages and the satellite transceivers. Initial instrumentation packages include microspectrometers, soil moisture probes, and various meteorological gauges. In 2002, the sensorweb will be redeployed at remote sites in Saskatchewan and Manitoba and the full access/control system will be tested through the Integrated Earth Sensing Workstation (IESW) in Ottawa.

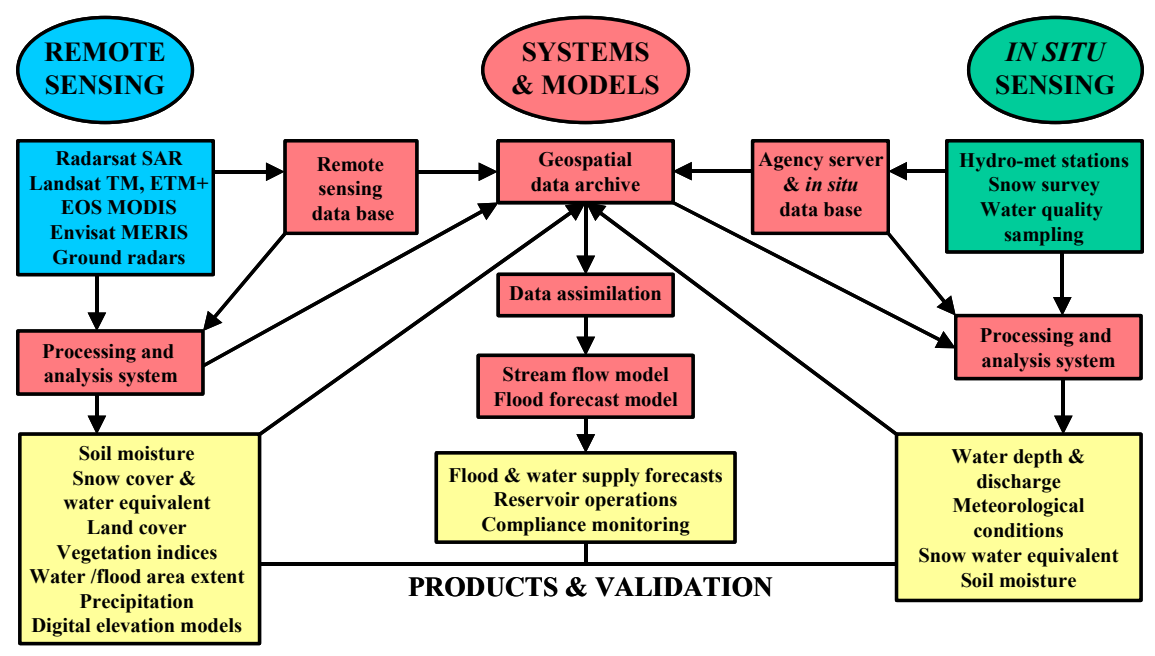

Figure 4. Example of the in situ sensor measurement assimilation framework in the context of watershed management (Teillet et al., 2001b).

\footnotetext{
${ }^{2}$ IGOS, http://www.igospartners.org/
} 


\subsection{Objective 2: Investigate Spatial Scale Issues}

The spatial distribution of the nodes in the sensorweb will be a critical variable in the context of a specific utilization. In future years, various spatial patterns will be explored for different land cover types and the data will be assimilated with simulations made from satellite image data at various spatial scales. It is hoped to determine the optimum spatial distribution of the sensorweb nodes as a function of spatial frequency in the land cover variable being measured and the number of nodes available.

\subsection{Objective 3: Investigate Measurement Accuracy Issues}

The availability of independent benchmark measurements for the sensorweb observations will allow an evaluation of the accuracy of the sensorweb instrumentation. Various statistical tests will be performed to determine the optimum method for computing means and moments from multi-node measurements of a given variable.

\subsection{Objective 4: Investigate Environmental Condition Issues}

The acquisition of datasets in a variety of environmental conditions throughout the season will allow an evaluation of the performance specifications of each component.

\subsection{Objective 5: Experimental Digital Infrastructure for Field and In-situ Campaigns and Equipment (EDIFICE)}

During the first two years of the program, ProWISE also plans to test and demonstrate remote webcam operations and telepresence at remote field sites. This initiative will go a long way to achieving the benefits of sensorweb deployments.

\subsection{Field Trials}

During the summer of 2002, a test deployment was made at Bratt's Lake Atmospheric Radiation Observatory (BLARO) in Saskatchewan, in collaboration with the Meteorological Service of Canada (MSC). As shown in Figure 5, the test configuration consisted of a compact mast with sensors recording temperature, relative humidity, downwelling solar radiation, rainfall, wind direction, wind speed, soil temperature, and soil moisture. The signals from all these sensors were wirelessly relayed to a central hub that then transmitted the data via satellite to the IESW in Ottawa. A more elaborate test deployment is planned for later in 2002 in the context of a flood forecasting application in Manitoba.

The initial prototype test-bed consists of a small sensorweb with five or six nodes and a local hub. Different wireless telecommunication strategies are being examined. Access and control will be remotely operated and will be tested from the individual nodes to the IESW as well as from the nodes to the hub and then to the IESW. Intelligent remote control of the microsensors is achieved through embedded systems specifically tailored to the geospatial application being investigated in the context of the framework discussed previously. Initially, there will be no internode communication. This is planned for a future phase of the sensorweb development.

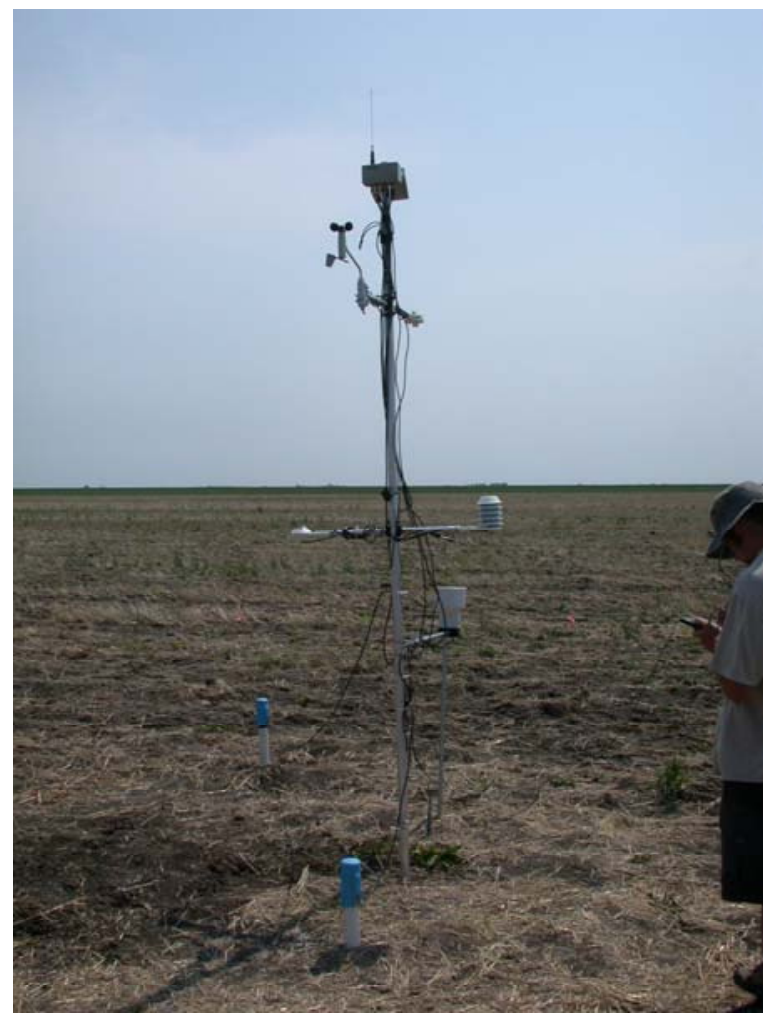

Figure 5. Fully instrumented wireless station deployed in Saskatchewan.

\section{CLOSING THE GAP}

The advanced technologies of today make it possible to develop integrated approaches to Earth sensing that encompass both remote and in situ sensing. In situ sensor measurement assimilation activities have the potential to significantly reduce the gap between partially validated information derived from remote sensing and quantitative geophysical and biospheric information needed to contribute to issues that society and policy-makers are striving to address. Such an effort will capitalize on the rapidly maturing technologies of micro-sensors and wireless telecommunications to expand the scope of remote sensing and make it more viable by providing solid underpinnings for the quality, robustness, and reliability of the information derived. It is from this perspective that integrated Earth sensing will help to make it possible for Earth observation technology to "cross the chasm" and be adopted by mainstream users of technology (Moore, 1991). 


\section{REFERENCES}

Buxton, W. (1997). Living in Augmented Reality: Ubiquitous Media and Reactive Environments. In Video Mediated Communication, K. Finn, A. Sellen \& S. Wilber (Eds.). Hillsdale, N.J.: Lawrence Erlbaum Assoc, ISBN 0805822887, pp. 363-384.

Delin, K.A., and Jackson, S.P. (2000). "Sensor Web for In-situ Exploration of Gaseous Biosignatures", Proceedings of 2000 IEEE Aerospace Conference, Big Sky, Montana, March 2000, 8 pages. (See also http://sensorwebs.jpl.nasa.gov/resources/DelinJackson2000.pdf)

Delin, K.A., and Jackson, S.P. (2001). "The Sensor Web: A New Instrument Concept", Proceedings of SPIE's Symposium on Integrated Optics, San Jose, California, January 2001, 9 pages. (See also http://sensorwebs.jpl.nasa.gov/resources/sensorwebconcept.pdf)

Hochheim, K.P., Hirose, T., Bennett, J., Bullock, P.R., Raddatz, R.L., Fedosejevs, G., and Teillet, P.M. (2002). "Improved Crop Specific Projections from the Integration of In Situ and Remote Sensing Data in an Agrometeorological Model", Proceedings of the 2002 IEEE Geoscience and Remote Sensing Symposium (IGARSS 2002) and the 24th Canadian Symposium on Remote Sensing, Toronto, Ontario, Volume IV, pp. 2391-2393, also on CD-ROM.

Hollar, S. E.-A. (2000). COTS Dust, M.Sc. Thesis, University of California, Berkeley, CA 94720, USA, 90 pages.

Justice, C.O., and Townshend, J.R.G. (1981). "Integrating ground data with remote sensing", In Terrain Analysis and Remote Sensing, J.R.G. Townshend (Ed.), pp. 38-58, Chapter 3, George Allen \& Unwin, London.

Lee, K. (1975). "Ground Investigations in Support of Remote Sensing", In Manual of Remote Sensing, R.G. Reeves (Ed.), pp. 805-856. American Society of Photogrammetry, Virginia.

Mann, S. (1997). "Wearable Computing: A First Step Toward Personal Imaging", Computer, 30(2), February 1997 (See also http://wearcam.org/ieeecomputer/r2025.htm)

Moore, G.A. (1991). Crossing the Chasm: Marketing and Selling Technology Products to Mainstream Consumers, Published by Harper Business, a Division of Harper Collins Publishers. ISBN 0-88730-519-9, 223 pages.
NASA (2000). Exploring Our Home Planet: Earth Science Enterprise Strategic Plan, NASA Headquarters, Washington, DC 20546.

Pettinger, L.R. (1971). "Field Data Collection - An Essential Element in Remote Sensing Applications", Proceedings of the International Workshop on Earth Resources Survey Systems, Washington, DC, pp. 49-64.

Pister, K.S.J., Kahn, J.M., and Boser, B.E. (1999) "Smart Dust: Wireless Networks of Millimeter-Scale Sensor Nodes", Highlight Article in 1999 Electronics Research Laboratory Research Summary, Department of Electrical Engineering and Computer Science, University of California, Berkeley, CA 94720.

Teillet, P.M. (1995). "The Role of Surface Observations in Support of Remote Sensing", In Canadian Remote Sensing Contributions to Understanding Global Change, E. LeDrew, M. Strome and F. Hegyi (Eds.), Department of Geography Publication Series, No. 38, University of Waterloo, Ontario, pp. 333-351, Chapter 17, and pp. 408415 References.

Teillet, P.M., Thome, K.J., Fox, N., and Morisette, J.T. (2001a). "Earth Observation Sensor Calibration Using A Global Instrumented and Automated Network of Test Sites (GIANTS)", Proceedings of SPIE Conference 4550 on Sensors, Systems, and Next-Generation Satellites V, Toulouse, France, Eds. H. Fujisada, J.B. Lurie, and K. Weber, SPIE Volume 4550, pp. 246-254.

Teillet, P.M., Dudelzak, A.E., Pultz, T.J., McNairn, H., and Chichagov, A. (2001b). "A Framework for In-Situ Sensor Measurement Assimilation in Remote Sensing", Proceedings of the $23^{\text {rd }}$ Canadian Symposium on Remote Sensing, Québec City, Québec, 21-24 August, pp. 111118.

Wood, M.D., Henderson, I., Pultz, T.J., Teillet, P.M., Zakrevsky, J.G., Crookshank, N., Cranton, J., and Jeena, A. (2002). "Integration of Remote and In Situ Data: Prototype Flood Information Management System", Proceedings of the 2002 IEEE Geoscience and Remote Sensing Symposium (IGARSS 2002) and the 24th Canadian Symposium on Remote Sensing, Toronto, Ontario, Volume III, pp. 1694-1696, also on CD-ROM. 